\title{
Effects of prematuration culture with a phosphodiesterase-3 inhibitor on oocyte morphology and embryo quality in in vitro maturation
}

\author{
Mohammed Ashraf Cheruveetil ', Prasanna Kumar Shetty ${ }^{2}$, Arya Rajendran ${ }^{1}$, Muhammed Asif ${ }^{3}$, Kamini A Rao $^{1}$ \\ ${ }^{1}$ Milann Fertility Center, Bengaluru; ${ }^{2}$ KSHEMA IVF Fertility and Reproductive Medicine Center, Nitte University, Deralakatte, Mangaluru; ${ }^{3}$ Garbhagudi IVF \\ Center, Bengaluru, India
}

Objective: The study assessed the developmental potential of germinal vesicle (GV) oocytes subjected to in vitro maturation (IVM) after prematuration culture with cilostamide (a phosphodiesterase-3 inhibitor) and the impact of cilostamide exposure on the morphology of meiosis II (MII) oocytes and subsequent embryo quality.

Methods: In total, 994 oocytes were collected from 63 patients. Among 307 GV oocytes, 140 oocytes were selected for the experimental group and 130 oocytes for the control group. The denuded GV-stage oocytes were cultured for 6 hours with cilostamide in the experimental group and without cilostamide in the control group. After 6 hours, the oocytes in the experimental group were washed and transferred to fresh IVM medium. The maturational status of the oocytes in both groups was examined at 26, 36 , and 48 hours. Fertilization was assessed at 18 hours post-intracytoplasmic sperm injection. Embryo quality was assessed on days 3 and 5 .

Results: In total, $92.1 \%$ of the oocytes remained in the GV stage, while $6.4 \%$ converted to the MI stage $(p<0.01)$ after cilostamide exposure. In both groups, more Mll oocytes were observed at 36 hours ( $25.8 \%$ vs. $21.5 \%)$ than at 26 hours $(10.8 \%$ vs. $14.6 \%)$ and 48 hours $(13 \%$ vs. $7.9 \%)(p>0.05)$. With the advent of cilostamide, blastocyst quality was better in the experimental group than in the control group $(p<0.05)$. Conclusion: Cilostamide effectively blocked nuclear maturation and promoted cytoplasmic growth. Prematuration culture with cilostamide enabled synchronization between cytoplasmic and nuclear maturity, resulting in better blastocyst outcomes.

Keywords: In vitro maturation; Intracytoplasmic sperm injection; Germinal vesicle oocyte; Phosphodiesterase-3 inhibitor

\section{Introduction}

The term in vitro maturation (IVM) refers to the maturation of immature oocytes retrieved from antral follicles with minimal or no gonadotropin stimulation [1]. Developed as early as 1935 by Pincus and Enzmann, this technique—although it seemed promising — did not come to the limelight like conventional in vitro fertilization (IVF). IVM

Received: February 8, 2021 - Revised: May 19, 2021 · Accepted: May 25, 2021 Corresponding author: Mohammed Ashraf Cheruveetil Milann Fertility Center, Bengaluru 560001, India

Tel: +91-96-6381-7857 Fax: +91-80-2201-1333 E-mail: mashraf.emb@gmail.com

This is an Open Access article distributed under the terms of the Creative Commons Attribution Non-Commercial License (http://creativecommons.org/licenses/by-nc/4.0/) which permits unrestricted non-commercial use, distribution, and reproduction in any medium, provided the original work is properly cited. was initially done in natural cycles and with the advent of better protocols, the option of follicle-stimulating hormone (FSH) priming along with luteinizing hormone $(\mathrm{LH}) /$ human chorionic gonadotrophin (hCG) triggering has been advocated to enhance oocyte competence [2]. Irrespective of FSH/LH/hCG priming, the outcomes of IVM for oocytes with regard to fertilization/implantation rates have been bleak in comparison with conventional stimulation cycles [3]. The suboptimal embryo quality obtained from IVM oocytes could be due to asynchrony between nuclear and cytoplasmic maturation at the cellular level [4]. Nuclear maturation comprises re-initiation of meiosis in oocytes arrested at prophase I, which is heralded by the loss of the nucleolar membrane (germinal vesicle [GV] breakdown) and ends in the completion of meiosis I with a concomitant expulsion of the first polar body [5]. Cytoplasmic maturation encompasses 
all the events at the organelle level along with various epigenetic modifications that prepare oocytes for subsequent fertilization and embryo development [6]. Furthermore, cytoplasmic maturation cannot be quantified by events as nuclear maturation and is therefore poorly understood. One approach that has been postulated to enhance oocyte competence is to promote nuclear and cytoplasmic synchrony by delaying nuclear maturation while simultaneously allowing cytoplasmic maturation to manifest [7]. This might possibly lead to the development of good-quality embryos. In vivo, cyclic adenosine monophosphate (cAMP) is the main meiotic inhibitor that keeps the oocyte in an arrested state (prophase I) until the hours preceding ovulation. The CAMP generated in granulosa cells is also transported via gap junctions $[8,9]$. The maintenance of an elevated intra-oocyte CAMP level maintains meiotic arrest and keeps the oocyte in its dormant state. Synchrony can therefore be achieved by a prematuration culture that maintains higher levels of CAMP and inhibits the meiotic cycle within the oocyte in vivo $[10,11]$. Levels of CAMP are maintained by inhibiting the enzyme-phosphodiesterase (PDE) or by upregulating adenyl cyclase. Although nuclear and cytoplasmic maturation are stepwise processes, their coordination is essential for the development of competent oocytes. Various studies have shown that about $5 \%$ to $20 \%$ of oocytes retrieved in controlled ovarian stimulation (COS) cycles are immature $[1,12]$. Some of these oocytes have the potential for spontaneous maturation during in vitro culture, and are used for intracytoplasmic sperm injection (ICSI). However, immature oocytes are usually discarded due to the possibility of an increased rate of miscarriages or risk of anomalies $[13,14]$. In patients with a cohort of asynchronized follicles, where there is a higher proportion of immature oocytes, the use of these oocytes is essential to increase the number of injectable oocytes for ICSI and eventually to generate more embryos for transfer. Studies have explored various prematuration cultures of immature oocytes, probing the use of PDE-3 inhibitors (PDE-3Is) at an ideal concentration and duration of exposure [15]. The main challenge in developing IVM culture systems is to adopt the ideal culture conditions required in a particular stage of oocyte dependency [16]. PDE-3 is located in oocytes and PDE-4 in granulosa cells. Hence, the PDE-3I cilostamide is unique as it exerts its effects on oocytes specifically and has no effect on granulosa cells $[17,18]$. Hence, it causes a delay in nuclear maturation without hindering cytoplasmic maturation [19]. The objective of this study was to assess the developmental potential of GV oocytes aspirated in COS cycles that were subjected to IVM after prematuration culture with cilostamide. We also attempted to study the impact of cilostamide exposure at the GV stage on the morphology of meiosis II (MII) oocytes and subsequent embryo quality.

\section{Methods}

\section{Ethical approval}

The study was conducted at a tertiary care reproductive medicine unit in India. It was approved by the Institutional Review Board and the Nitte Central Ethics Committee (NU/CEC/2018/0191 NU/CEC/ 2020/2069). Informed consent was obtained from patients prior to the laboratory access of oocytes and sperm.

\section{Study population and oocyte source}

In total, 994 oocytes were collected from 63 patients, of which 307 were GV oocytes. Thirty-seven morphologically abnormal GV oocytes were excluded from the study. The present study comprised 270 viable GV oocytes obtained from women aged $25-35$ years, who underwent COS for IVF/ICSI at Milann Fertility Center from April 2018 to March 2020. Patients with a minimum of four GV oocytes were included in the study. Only one treatment cycle per patient was incorporated in the study.

\section{Stimulation protocol}

COS was started on day 2 or 3 of the menstrual cycle after a baseline transvaginal ultrasound scan (Voluson $\mathrm{P} 6$ with a 4-8 MHz vaginal probe) and hormonal assessment (estradiol $\left[\mathrm{E}_{2}\right]$, progesterone, and LH if deemed necessary). Gonadotropins included either recombinant FSH, (Recagon, Organon, Oss, The Netherlands; Gonal F, Merck Serono, Modugno, Italy) or human menopausal gonadotropin (Menopur; Ferring Leciva, As, Czech Republic). The starting dose was calculated based on age, body mass index, and antral follicle count, and ranged from 112.5 to $187.5 \mathrm{IU}$ daily for 4 days. The ovarian response to stimulation was monitored on the fifth day of stimulation with transvaginal ultrasound scan and serum $\mathrm{E}_{2}, \mathrm{LH}$, and progesterone measurements, and the dose of gonadotropins was adjusted accordingly. A gonadotropin-releasing hormone antagonist $(0.25 \mathrm{mg}$; Cetrotide, Merck, Darmstadt, Germany) was given daily subcutaneously based on a flexible antagonist protocol when the dominant follicle was $\geq 12-14 \mathrm{~mm}$ in diameter or the serum $\mathrm{E}_{2}$ level was $>350-400$ $\mathrm{pg} / \mathrm{mL}$ until the day of triggering. Triggering was performed when at least 3 follicles reached $\geq 17 \mathrm{~mm}$ in diameter. Oocytes were aspirated transvaginally $35-36$ hours post-triggering under intravenous sedation and ultrasound guidance. A single-lumen oocyte retrieval needle (Vitrolife, Vastra Frolunda, Sweden) was used.

\section{Laboratory protocol}

\section{1) Preparation of cilostamide}

The PDE-3I cilostamide (Cayman Chemical, Ann Arbor, MI, USA) was used for prematuration culture. A stock solution was prepared by dissolving cilostamide in crystalline form with dimethyl sulfoxide. 
A final concentration of $1 \mu \mathrm{M}$ cilostamide was added to the prematuration culture with the intention of achieving efficient meiotic inhibition and maximum reversibility of inhibition.

\section{2) Preparation of cumulus-oocyte complexes}

Cumulus-oocyte complexes (COCs) were incubated in the culture medium (G-IVF-PLUS, Vitrolife) covered with mineral oil (Ovoil, Vitrolife) at $37^{\circ} \mathrm{C}$ in $6 \% \mathrm{CO}_{2}$ for $2-3$ hours. Cumulus cells were removed after exposure to G-Mops (3-N-morpholino-propane sulfonic acid) buffered medium containing $80 \mathrm{lU} / \mathrm{mL}$ hyaluronidase (Hyase, Vitrolife) for 30 seconds with the help of a glass Pasteur pipette (Humagen Fertility Diagnostics, Charlottesville, VA, USA). The nuclear status of the denuded oocytes was then assessed. Oocytes that were observed to have released the first polar body were considered mature and used for ICSI. Immature oocytes with a prominent nucleus (GVs) were assigned to the study and taken up for the prematuration culture.

\section{Experimental setup}

\section{1) Prematuration culture}

The denuded GV-stage oocytes of each patient were distributed into two groups (experimental and control group) and subjected to two different culture conditions. All immature oocytes were cultured individually in $25-\mu \mathrm{L}$ drops of medium overlaid with oil in a humidified atmosphere at $37^{\circ} \mathrm{C}$ in $6 \% \mathrm{CO}_{2}$. The oocytes in the experimental group were placed in an IVM medium (Oocyte Maturation Medium; Cooper Surgical/SAGE, Trumbull, CT, USA) supplemented with $5 \mathrm{mg} /$ $\mathrm{mL}$ serum protein substitute and FSH/LH $(75 \mathrm{mlU} / \mathrm{mL}$ for each; Serono, Mississauga, ON, Canada) supplemented with $1 \mu \mathrm{M}$ cilostamide, while the oocytes in the control group were placed in an IVM medium without cilostamide. After culturing these GV-stage oocytes for 6 hours [20], the cilostamide was removed from the experimental group. These oocytes were then transferred to inhibitor-free IVM medium and cultured for 42 hours.

\section{2) Maturation of oocytes}

The oocytes were then classified as GV, MI, or MII. ICSI was performed on MIl oocytes, and the GV oocytes were cultured up to 48 hours.

\section{3) Semen samples for ICSI}

Semen samples were collected at the time of oocyte retrieval. After liquefaction, a simple preparative method, such as the swim-up or double density gradient method, was used to prepare semen samples for the ICSI procedures [21,22]. Since the oocytes did not mature synchronously during culture, the semen samples were frozen in multiple vials and thawed accordingly for further insemination.

\section{4) Intracytoplasmic sperm injection}

Morphologically normal spermatozoa were injected into the GV-converted MII oocytes. Following ICSI, these two groups (experimental and control groups) of injected oocytes were then incubated in $25-\mu \mathrm{L}$ drops of single-step medium (GTL; Vitrolife) for further development.

\section{5) Fertilization assessment}

Fertilization was assessed at 18 hours post-ICSI. Fertilization was determined by the appearance of two distinct pronuclei and two polar bodies.

\section{6) Embryo culture system}

The fertilized oocytes were cultured for 120 hours. Embryo development was observed at 24-hour intervals, which were continued until day 5 ( 120 hours). On days 3 and 5 , the embryos were scored as per the Istanbul consensus [23]. Embryo grading was performed and validated by expert embryologists.

\section{Statistical analysis}

Data were analyzed using IBM SPSS ver. 23 (IBM Corp., Armonk, NY, USA). The differences between the treatment groups were analyzed using the chi-square test and when appropriate, the two-tailed Fisher exact test. A $p$-value $<0.05$ was considered to indicate statistical significance.

\section{Results}

The present study comprised 270 GV oocytes, of which 140 oocytes were selected for the experimental group and 130 oocytes for the control group (Figure 1). The quality and morphology of the GV oocytes were similar in both the experimental and control groups $(p>0.05)$.

\section{Blocking of nuclear maturation by prematuration culture with cilostamide}

The GV oocytes in the experimental group were exposed to $1 \mu \mathrm{M}$ cilostamide for 6 hours. The control group was free from cilostamide. After 6 hours of culture in cilostamide, it was observed that $92.1 \%$ of oocytes remained in the GV stage and only $6.4 \%$ of oocytes had converted to the Ml stage. However, in the control group, 20.8\% of GV oocytes converted to the MI stage. A statistically significant result was observed between the experimental and control groups $(p<0.01)$ (Table 1). It was shown that cilostamide was effective at blocking nuclear maturation and promoting cytoplasmic growth. The number of $\mathrm{Ml}$ oocytes showed a statistically significant difference between the experimental and control groups $(p<0.001)$ (Table 1). 
2. Culture system of GV oocytes after 6 hours: progression of meiosis at 26, 36, and 48 hours in oocytes subjected to prematuration culture with cilostamide ( $1 \mu \mathrm{M})$ followed by IVM culture

The GV oocytes were then cultured in IVM medium (without cilostamide) and were observed at 26, 36, and 48 hours (Table 2). The experimental and the control groups showed no significant differences at 36 hours and 48 hours, although a significant difference was found for GV oocytes at 26 hours ( $p=0.05$ ). The experimental group showed the highest number of MII oocytes at 36 hours (25.8\%), followed by 48 hours (13\%) and 26 hours (10.8\%). The control group

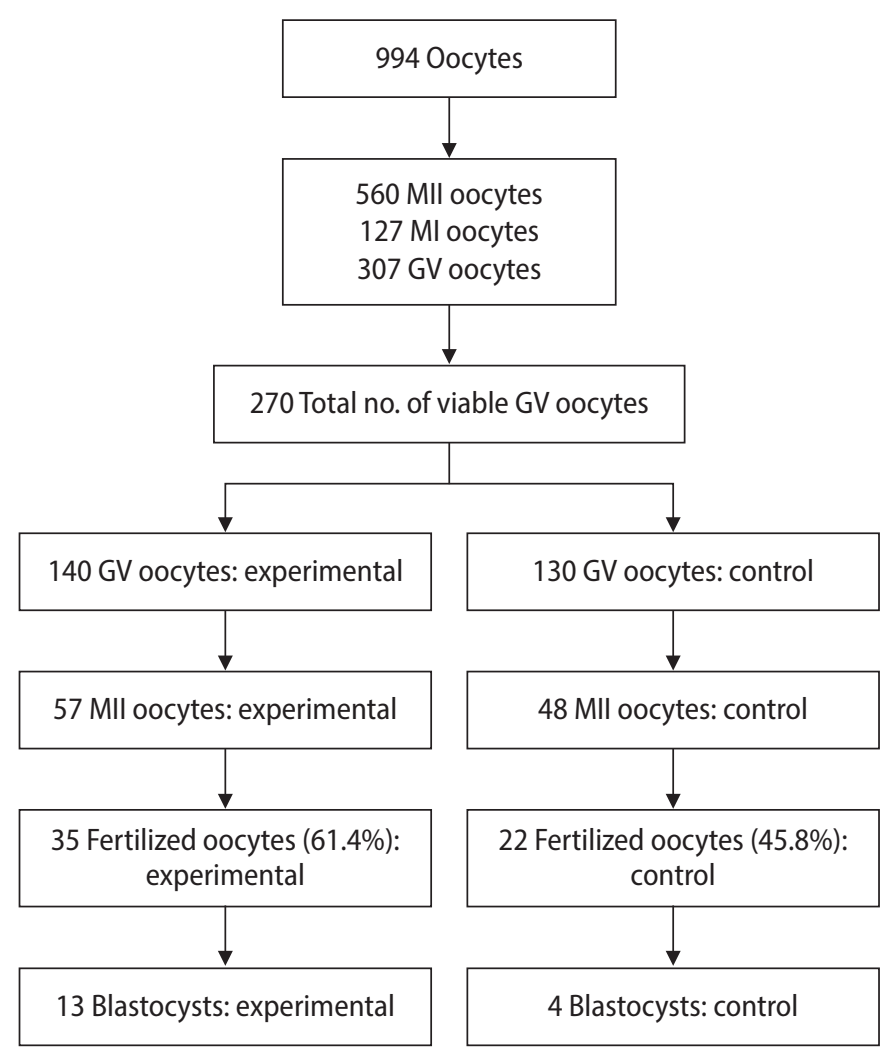

Figure 1. Distribution of oocytes retrieved and subsequent fertilization and embryo development. MII, meiosis II; MI, meiosis I; GV, germinal vesicle. also showed the maximum number of MIl oocytes at 36 hours (21.5\%), followed by 26 hours (14.6\%) and 48 hours (7.9\%). There was no statistically significant difference between the experimental and control groups $(p>0.05)$. For the 57 oocytes that matured from $140 \mathrm{GV}$ oocytes in the experimental group and the 48 oocytes that matured out of $130 \mathrm{GV}$ oocytes in the control group, the maturation rate was $41 \%$ and $37 \%$, respectively. The present study also observed an increased number of degenerated oocytes at 48 hours in the control group as compared to the experimental group (Table 2).

\section{Morphological assessment of MIl oocytes}

Cytoplasmic abnormalities such as increased granulations, the presence of vacuoles, refractile bodies, smooth endoplasmic reticulum clusters, organelle clustering, and dark cytoplasm were found in the MII oocytes of both the experimental and control groups (Figure 2). However, the morphological features were similar in both groups $(p>0.05)$. All the Mll oocytes were then injected with morphologically normal sperm.

\section{Fertilization of oocytes}

The total number of MII oocytes obtained was 57 in the experimental group and 48 in the control group. Fertilization was confirmed with the appearance of two distinct pronuclei and two polar bodies in 35 oocytes (61.4\%) in the experimental group and 22 oocytes (45.8\%) in the control group. However, there were no statistically sig-

Table 1. Efficacy of cilostamide $(1 \mu \mathrm{M})$ in maintaining the meiotic arrest of GV oocytes in the experimental group as compared to the control group

\begin{tabular}{lccc}
\hline Oocyte & $\begin{array}{c}\text { Experimental } \\
\left.\text { group }(\mathrm{n}=140)^{\mathrm{a}}\right)\end{array}$ & $\begin{array}{c}\text { Control group } \\
\left.(\mathrm{n}=130)^{\mathrm{b}}\right)\end{array}$ & $p$-value \\
\hline GV & $129(92.1)$ & $103(79.2)$ & $<0.01$ \\
Ml & $9(6.4)$ & $27(20.8)$ & $<0.001$ \\
Degenerated & $2(1.4)$ & 0 & 0.1 \\
\hline
\end{tabular}

Values are presented as number (\%). The $p<0.05$ is considered to indicate statistical significance.

GV, germinal vesicle; Ml, meiosis I.

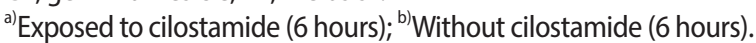

Table 2. Progression of meiosis at 26,36 , and 48 hours of IVM culture of oocytes subjected to prematuration culture with cilostamide (1 $\mu \mathrm{M})$ followed by IVM culture

\begin{tabular}{|c|c|c|c|c|c|c|c|c|c|c|c|c|}
\hline \multirow{2}{*}{ Variable } & \multicolumn{2}{|c|}{ GV } & \multirow{2}{*}{$p$-value } & \multicolumn{2}{|c|}{ MI } & \multirow{2}{*}{$p$-value } & \multicolumn{2}{|c|}{ MII } & \multirow{2}{*}{$p$-value } & \multicolumn{2}{|c|}{ Degenerated } & \multirow{2}{*}{$p$-value } \\
\hline & $E$ & C & & $E$ & C & & $E$ & C & & $E$ & $\mathrm{C}$ & \\
\hline After $26 \mathrm{hr}(\mathrm{E}=138, \mathrm{C}=130)$ & $103(74.6)$ & $83(63.8)$ & 0.05 & $17(12.3)$ & $24(18.5)$ & 0.1 & $15(10.8)$ & $19(14.6)$ & 0.3 & $3(2.2)$ & $4(3)$ & 0.6 \\
\hline After $36 \mathrm{hr}(E=120, C=107)$ & $61(50.8)$ & $49(45.8)$ & 0.4 & $23(19.1)$ & $27(25.2)$ & 0.2 & $31(25.8)$ & $23(21.5)$ & 0.4 & $5(4.2)$ & $8(7.5)$ & 0.2 \\
\hline After $48 \mathrm{hr}(\mathrm{E}=84, \mathrm{C}=76)$ & $36(42.9)$ & $34(44.7)$ & 0.8 & $28(33.3)$ & $25(32.9)$ & 0.9 & $11(13)$ & $6(7.9)$ & 0.2 & $9(10.7)$ & $11(14.5)$ & 0.4 \\
\hline
\end{tabular}

Values are presented as number (\%).

IVM, in vitro maturation; GV, germinal vesicle; MI, meiosis I; MII, meiosis II; E, experimental group; C, control group. 
nificant differences between the two groups ( $p>0.05$ ) (Figure 1).

\section{Cleavage of embryos}

The fertilized oocytes in the experimental $(n=35)$ and control $(n=22)$ groups were further cultured. After 27 hours, the embryo culture showed a higher frequency of cleavage in the experimental group (88.6\%) than in the control group (77.3\%), although the results were statistically insignificant $(p=0.2)$. Similar results were observed on day 2 and day 3 of embryo culture with regard to cleavage $(p>0.05)$. However, optimal cleavage was more frequent in the experimental group (40\%) than in the control group (27.3\%), although this difference did not reach statistical significance $(p=0.33$ ) (Table 3).

Even though the cleavage rate was similar in both groups, the proportion of good-quality embryos (i.e., those with no apparent morphological abnormalities and few anucleate fragments) obtained was higher in the experimental group than in the control group.

The experimental group showed the highest number of grade 1 embryos, followed by grade 2 and grade 3, whereas the control group showed the highest number of grade 2 embryos, followed by grade 1 and grade 3 . However, the distribution of grades 1,2 , and 3 among the experimental and control groups was similar $(p>0.05)$ (Figure 3).

\section{Hourly cleavage status of embryos}

The number of good-quality embryos was higher in the experimental group than in the control group, although this difference did not reach statistical significance. Considering the optimal time required to reach the cleavage stage, culturing for 26 hours and 36 hours resulted in the maximum number of embryos, whereas prolonged culture up to 48 hours did not give any advantage in terms of grade 1 embryos (Table 4).

Figure 4 shows the results of the development of human blastocysts from IVM GV oocytes. The present study yielded a total of 13 blastocysts in the experimental group and 4 blastocysts in the con-

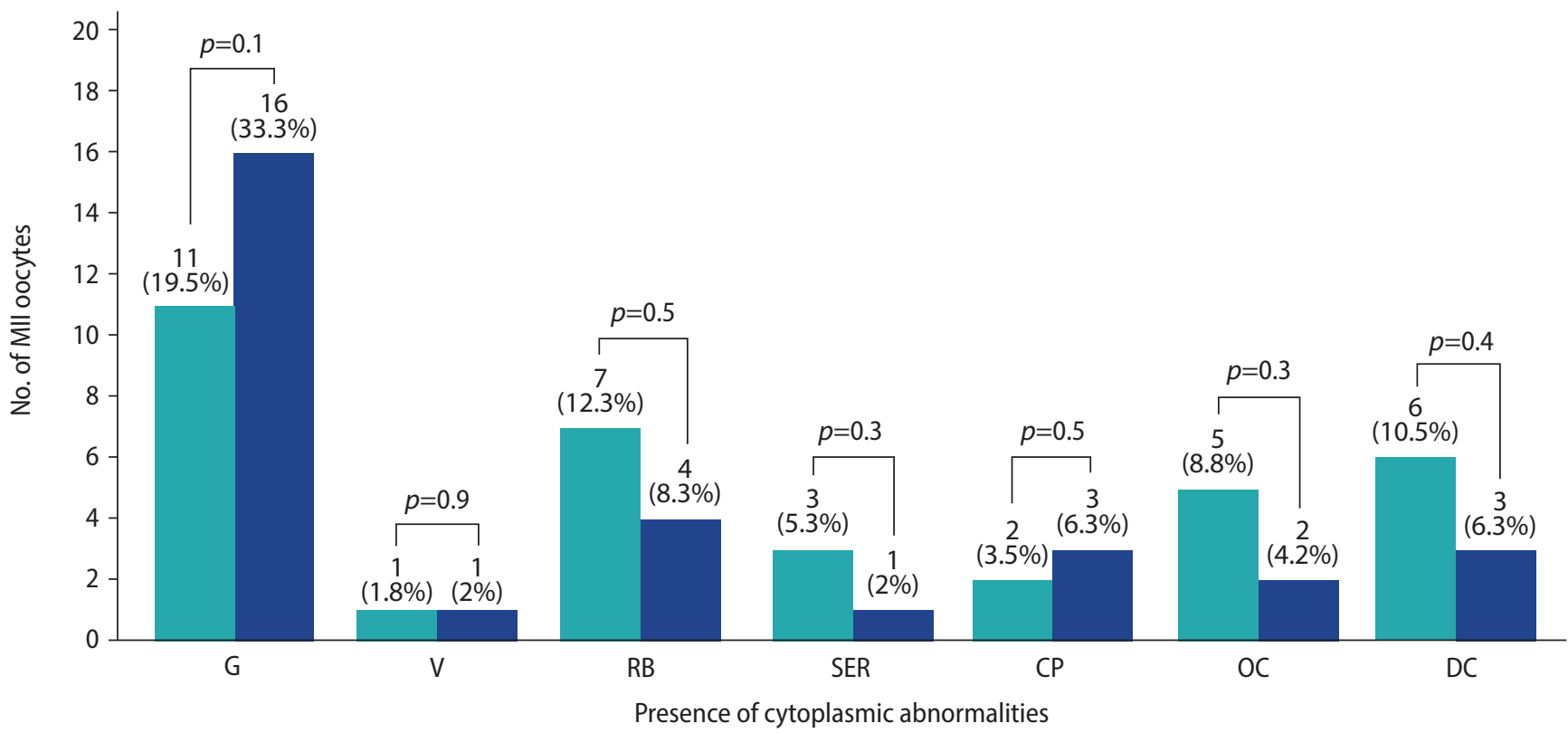

Figure 2. Morphological evaluation of cytoplasmic abnormalities of mature oocytes following in vitro maturation culture (meiosis II [MII]). G, granulations; $\mathrm{V}$, vacuoles; $\mathrm{RB}$, refractile bodies; $\mathrm{SER}$, smooth endoplasmic reticulum clusters; $\mathrm{CP}$, central pitting; $\mathrm{OC}$, organelle clustering; $\mathrm{DC}$, dark cytoplasm.

Table 3. Cleavage status of injected MII oocytes cultured in IVM medium

\begin{tabular}{|c|c|c|c|c|c|}
\hline \multirow{2}{*}{ Cleaved embryo } & \multicolumn{2}{|c|}{ Experimental $(n=35)$} & \multicolumn{2}{|c|}{ Control $(n=22)$} & \multirow{2}{*}{$p$-value } \\
\hline & No. of fertilized oocytes (\%) & Arrested & No. of fertilized oocytes (\%) & Arrested & \\
\hline No. of cleaved 2 PN (> $27 \mathrm{hr}$ ) & $31(88.6)$ & 4 & $17(77.3)$ & 5 & 0.26 \\
\hline No. of 4-cell embryos on day 2 & $28(80)$ & 3 & $13(59.1)$ & 4 & 0.09 \\
\hline No. of 8-cell embryos on day 3 & $23(65.7)$ & 5 & $9(40.9)$ & 4 & 0.07 \\
\hline No. of embryos with optimal cleavage & $14(40)$ & & $6(27.3)$ & & 0.33 \\
\hline
\end{tabular}

MII, meiosis II; IVM, in vitro maturation; PN, pronuclei. 
Table 4. Embryonic development of MII oocytes on day 3 subjected to prematuration culture with cilostamide (1 $\mu$ M) followed by IVM culture

\begin{tabular}{|c|c|c|c|c|c|c|c|c|c|}
\hline \multirow{2}{*}{ Day 3 embryo grading } & \multicolumn{2}{|c|}{$26 \mathrm{hr}$} & \multirow{2}{*}{$p$-value } & \multicolumn{2}{|c|}{$36 \mathrm{hr}$} & \multirow{2}{*}{$p$-value } & \multicolumn{2}{|c|}{$48 \mathrm{hr}$} & \multirow{2}{*}{$p$-value } \\
\hline & $E(n=7)$ & $C(n=3)$ & & $E(n=12)$ & $C(n=5)$ & & $E(n=4)$ & $C(n=1)$ & \\
\hline $8 C G 1$ & $4(57.1)$ & $1(33.3)$ & 0.5 & $7(58.3)$ & $1(20)$ & 0.2 & 0 & 0 & 0 \\
\hline $8 C G 2$ & 2 (28.6) & $2(66.7)$ & 0.3 & $4(33.3)$ & $3(60)$ & 0.3 & $1(25)$ & 0 & 0.6 \\
\hline $8 C G 3$ & $1(14.3)$ & 0 & 0.5 & $1(8.3)$ & $1(20)$ & 0.5 & $3(75)$ & $1(100)$ & 0.6 \\
\hline
\end{tabular}

Values are presented as number (\%). Grade 1 (G1), good embryos with less than $10 \%$ fragmentation, equal blastomeres, and no multinucleation; grade 2 (G2), fair embryos with 10\%-25\% fragmentation; grade 3 (G3), poor embryos with severe fragmentation (>25\%), unequal blastomeres, and multinucleation. MII, meiosis II; IVM, in vitro maturation; E, experimental group; C, control group.

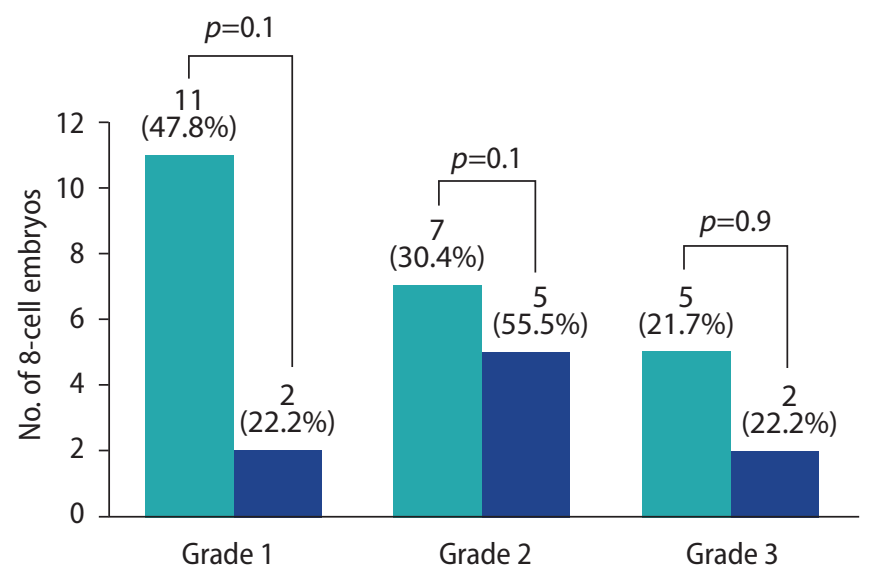

Figure 3. Grading of day 3 embryos (as per the Istanbul consensus) following in vitro maturation culture.

trol group (Table 5). Furthermore, we observed a statistically significant difference in the number of blastocysts obtained between the experimental and control groups $(p<0.05)$.

\section{Discussion}

The complexities around the mechanisms leading to final oocyte maturation remain largely unresolved, which may be why the technique of IVM lacks a precise rationale. The present study was based on the hypothesis that $\operatorname{COS}$ can lead to an acceleration of meiotic progression in oocytes, wherein the cytoplasmic maturation often lags behind nuclear maturation [24]. Studies have also shown that the extrusion of the polar body appears much earlier in denuded oocytes; this is deficient in cumulus cells due to the loss of gap junctions, which provide the oocyte with CAMP to maintain dormancy. Therefore, oocytes relieved from the inherent inhibitory effect on meiosis can proceed with nuclear maturation at a faster rate [25]. It has been shown that asynchrony between nuclear and cytoplasmic maturation can cause a negative impact on IVM outcomes. Pre-maturation culture has emerged as a potential method to prolong nucle-
Table 5. Development of blastocysts from MIl oocytes subjected to prematuration culture with cilostamide $(1 \mu \mathrm{M})$ followed by IVM culture

\begin{tabular}{|c|c|c|c|c|c|c|}
\hline \multirow[b]{2}{*}{ Variable } & \multicolumn{2}{|c|}{$26 \mathrm{hr}$} & \multicolumn{2}{|c|}{$36 \mathrm{hr}$} & \multicolumn{2}{|c|}{$48 \mathrm{hr}$} \\
\hline & $\begin{array}{c}E \\
(n=7)\end{array}$ & $\underset{(n=3)}{C}$ & $\begin{array}{c}E \\
(n=12)\end{array}$ & $\underset{(n=5)}{C}$ & $\begin{array}{c}E \\
(n=4)\end{array}$ & $\underset{(n=1)}{C}$ \\
\hline \multicolumn{7}{|l|}{ Grade } \\
\hline 1,1 & 1 & - & 1 & - & - & - \\
\hline 1,2 & 1 & - & 2 & - & - & - \\
\hline 2,1 & 1 & - & 1 & 1 & - & - \\
\hline 2,2 & 1 & 1 & 2 & - & - & - \\
\hline 3,2 & - & 1 & 1 & 1 & - & - \\
\hline 3,3 & - & - & - & - & 2 & - \\
\hline Total no. of blastocysts & 4 & 2 & 7 & 2 & 2 & nil \\
\hline \multicolumn{7}{|l|}{ Cell arrest } \\
\hline Compaction arrest & 1 & 1 & 2 & 1 & - & - \\
\hline 8 & 1 & - & 2 & 1 & 1 & 1 \\
\hline 9 & - & - & - & - & 1 & - \\
\hline 10 & 1 & - & 1 & - & - & - \\
\hline 12 & - & - & - & 1 & - & - \\
\hline
\end{tabular}

Blastocysts are graded according to the stage of development, using grades of the inner cell mass (1-3; good to poor) based on the number of cells, compaction, and adhesion, and grades of the trophectoderm (1-3; good to poor) based on the number of cells and the cohesiveness of the epithelium. MII, meiosis II; IVM, in vitro maturation; E, experimental group; C, control group.

ar maturation and also allows adequate cytoplasmic competence to manifest. Incomplete cytoplasmic maturity can result in multinucleation and aneuploidy, thereby impairing embryo development [8]. However, to date, no consensus exists on the IVM culture system or which, if any, additives are beneficial.

CAMP is the main meiotic inhibitor that keeps oocytes in an arrested state (prophase I) until the hours preceding ovulation. Intra-oocyte cAMP levels are dictated by a balance in the activity of adenylyl cyclase, which assists in the synthesis of CAMP, and PDE, which assists in the degradation of CAMP [26,27]. Furthermore, the cAMP generated in granulosa cells is transported via gap junctions [28,29]. The mainte- 

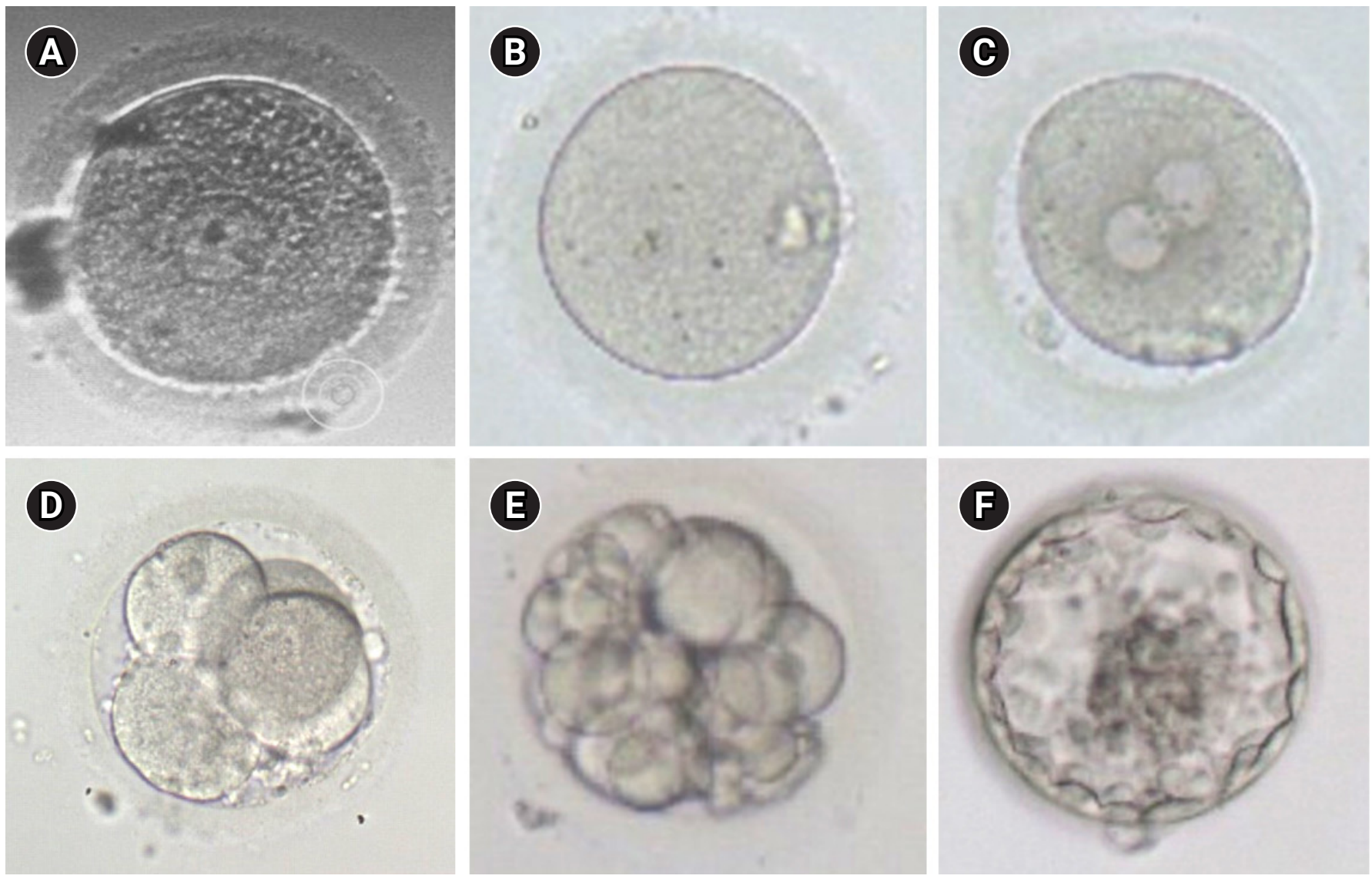

Figure 4. Schematic representation of the developmental stages of oocytes and embryos subjected to prematuration culture with cilostamide ( $1 \mu \mathrm{M}$ ) followed by in vitro maturation (IVM) culture. (A) Germinal vesicle oocyte during treatment with cilostamide (0-6 hours). (B) Metaphase II oocyte developed with IVM after cilostamide treatment. (C) Fertilized oocyte showing juxtaposed pronuclei and two polar bodies in the perivitelline space following IVM culture. (D, E) Day 2 and day 3 embryo development. (F) Blastocyst development following IVM culture.

nance of an elevated intra-oocyte cAMP level can keep meiosis in a dormant state. Hence, there is a delay in nuclear maturation, which allows time for cytoplasmic maturation. cAMP levels can be elevated within the oocyte with the help of kinase inhibitors (roscovitine or butyrolactone) [30] or by protein synthesis inhibitors (cycloheximide or puromycin) [31]. As these enzymes can act on both oocytes and granulosa cells, they may impair granulosa cell-oocyte interactions and future embryogenesis. Therefore, in the present study, we used the PDE3 I cilostamide, the action of which is oocyte-specific.

While using cilostamide, the reversibility of meiotic inhibition is an important consideration. A higher concentration of cilostamide can harm the reversibility of meiotic inhibition and spindle structure. Hence, unduly extending the period between transcriptional inactivation and initiation of meiotic maturation may reduce the developmental competence of immature oocytes [20]. Thus, in the present study we used denuded GV oocytes that were exposed to 6 hours of prematuration culture.

As compared to previous studies [32], the present study showed effectiveness in blocking nuclear maturation with $1 \mu \mathrm{M}$ cilostamide for 6 hours. There was a statistically significant increase in the number of oocytes that remained in the GV stage, as compared to those that progressed towards $\mathrm{M} 1$, between the experimental and control groups $(92.1 \%$ vs. $79.2 \%, p<0.01)$. At the end of meiotic arrest, a higher proportion of GV-stage oocytes with the surrounded-nucleolus chromatin configuration [33] indicates a morphological parameter of transcriptional repression. This transformation from a diffused or non-surrounded nucleolus configuration to a compacted or surrounded-nucleolus shape is crucial for successful embryonic development [34]. The results were comparable to those reported in previous studies [20,35]. In our study, we used SAGE medium (Cooper Surgical) to induce oocyte maturation. It has shown proven efficacy for maturing oocytes retrieved from patients with polycystic ovarian syndrome, as well as for the maturation of immature oocytes aspirated prior to treatment of ovarian malignancy [36,37].

The shorter duration of IVM culture is also an independent predictor of better oocyte and embryo quality. The outcome after with- 
drawal of the inhibitor is still a debatable topic. Son et al. [38] showed that embryos derived from late-maturing oocytes ( $>48$ hours of IVM) were of suboptimal quality in comparison with oocytes maturing between 24 hours and 30 hours of IVM. Therefore, it can be inferred that prolonged blockage of meiotic progression can result in poor-quality embryo outcomes. After the removal of cilostamide blockage, the GV oocytes were cultured in IVM medium and were observed at 26 hours, 36 hours, and 48 hours. A higher number of MII oocytes was observed at 36 hours in the experimental group (25.8\%) than in the control group (21.5\%). Previous studies have followed several approaches concerning the time of culture for IVM, but there has been no consensus on the optimal maturation time for GV oocytes in IVM cycles in the literature so far $[39,40]$. We found no significant difference in the fertilization rates among MII oocytes with or without cilostamide exposure $(p=0.3)$. However, the study by Nogueira et al. [32] showed an increased fertilization rate and the study by Jee et al. [20] reported a paradoxical reduction in the fertilization rate.

A previous study by Xie et al. [40] showed that the rates of cleavage and blastocyst development in oocytes maturing at 48 hours after IVM (experimental, 72.2\%; control, 19.0\%) were significantly lower than in GV oocytes matured 24 hours after IVM (experimental, 91.5\%; control, 50.4\%). The present study also showed comparable results after 48 hours of IVM culture. From a metabolic perspective, successful IVM of an immature oocyte results from the facilitation of an appropriate environment for the oocyte to attain its developmental competence. $\mathrm{COCs}$ require glucose in the medium for metabolism to take place. Cumulus cells possess a substantial capacity for metabolizing glucose, which further produces carboxylic acids and adenosine triphosphate (ATP). The tricarboxylic acid cycle utilizes carboxylic acids within the oocyte. The developmental competence of the oocyte during IVM determines the generation of ATP by the COC. ATP, which is generated by oxidative phosphorylation, also forms the substrate for the production of CAMP. Meiotic inhibition during follicle development is maintained by cAMP. Even in the absence of cumulus cells, immature oocytes can be matured in vitro by the projections of cumulus cells embedded in the zona pellucida. These projections of cumulus cells provide inhibitory and stimulatory signals to the oocytes [41]. Therefore, it is evident that cumulus-free immature human oocytes can mature in IVM medium and also have the potential for early embryonic (cleavage and blastocyst) development.

Furthermore, we observed a statistically insignificant increase in embryo grading in the experimental group, when compared to the control group. However, surprisingly, we found a statistically significant increase in blastocysts in the experimental group (22.8\% of MII oocytes injected) as compared to the control group (8.3\%). Hence, narrowing the nuclear-cytoplasmic asynchrony with cilostamide plays an instrumental role in obtaining more blastocysts from IVM GV oocytes and could be used as an effective tool in increasing oocyte competence during IVM culture.

In conclusion, the present study provides insights into the IVM culture system for maturing GV oocytes. The developmental competence of oocytes can be enhanced through an improved understanding of the mechanisms that regulate meiotic competence, arrest, and resumption. Further studies are needed to understand the mechanisms through which meiosis is regulated in human oocytes and how oocyte-derived factors influence zygotic genome activation and embryonic developmental competence in human oocytes.

\section{Conflict of interest}

No potential conflict of interest relevant to this article was reported.

\section{Acknowledgments}

We thank Dr. Mir Jaffar, Scientific Director, Milann Fertility Center, India for critical advice on this manuscript.

\section{Author contributions}

Conceptualization: MAC, PKS, KAR. Data curation: MAC, MA. Formal analysis: PKS, KAR. Methodology: MAC, PKS. Project administration: AR. Visualization: MAC, MA. Writing-original draft: MAC. Writing-review \& editing: MAC, AR, PKS, MA, KAR.

\section{References}

1. Cha KY, Chian RC. Maturation in vitro of immature human oocytes for clinical use. Hum Reprod Update 1998;4:103-20.

2. Practice Committees of the American Society for Reproductive Medicine and the Society for Assisted Reproductive Technology. In vitro maturation: a committee opinion. Fertil Steril 2013;99: 663-6.

3. U.S. Department of Health and Human Services Centers for Disease Control and Prevention. 2005 Assisted reproductive technology success rates: National summary and fertility clinic reports. Atlanta: U.S: Department of Health and Human Services Centers for Disease Control and Prevention; 2007.

4. Moor RM, Dai Y, Lee C, Fulka J Jr. Oocyte maturation and embryonic failure. Hum Reprod Update 1998;4:223-36.

5. Pan B, Li J. The art of oocyte meiotic arrest regulation. Reprod Biol Endocrinol 2019;17:1-12.

6. Bassham S, Canestro C, Postlethwait JH. Evolution of develop- 
mental roles of Pax2/5/8 paralogs after independent duplication in urochordate and vertebrate lineages. BMC Biol 2008;6:35.

7. Combelles CM, Cekleniak NA, Racowsky C, Albertini DF. Assessment of nuclear and cytoplasmic maturation in in-vitro matured human oocytes. Hum Reprod 2002;17:1006-16.

8. Conti M, Andersen CB, Richard FJ, Shitsukawa K, Tsafriri A. Role of cyclic nucleotide phosphodiesterases in resumption of meiosis. Mol Cell Endocrinol 1998;145:9-14.

9. Son WY, Chung JT, Demirtas E, Holzer H, Sylvestre C, Buckett W, et al. Comparison of in-vitro maturation cycles with and without in-vivo matured oocytes retrieved. Reprod Biomed Online 2008;17:59-67.

10. Ferrell JE Jr. Xenopus oocyte maturation: new lessons from a good egg. Bioessays 1999;21:833-42.

11. Taieb F, Thibier C, Jessus C. On cyclins, oocytes, and eggs. Mol Reprod Dev 1997;48:397-411.

12. Gardner DK, Weissman A, Howles CM, Shoham Z. Textbook of assisted reproductive technologies: laboratory and clinical perspectives. Boca Raton: CRC Press; 2008.

13. Shu Y, Gebhardt J, Watt J, Lyon J, Dasig D, Behr B. Fertilization, embryo development, and clinical outcome of immature oocytes from stimulated intracytoplasmic sperm injection cycles. Fertil Steril 2007;87:1022-7.

14. Alvarez C, Garcia-Garrido C, Taronger R, Gonzalez de Merlo G. In vitro maturation, fertilization, embryo development \& clinical outcome of human metaphase-l oocytes retrieved from stimulated intracytoplasmic sperm injection cycles. Indian J Med Res 2013;137:331-8.

15. Guimaraes AL, Pereira SA, Kussano NR, Dode MA. The effect of pre-maturation culture using phosphodiesterase type 3 inhibitor and insulin, transferrin and selenium on nuclear and cytoplasmic maturation of bovine oocytes. Zygote 2016;24:219-29.

16. Hardy K, Wright CS, Franks S, Winston RM. In vitro maturation of oocytes. Br Med Bull 2000;56:588-602.

17. Tsafriri A, Chun SY, Zhang R, Hsueh AJ, Conti M. Oocyte maturation involves compartmentalization and opposing changes of CAMP levels in follicular somatic and germ cells: studies using selective phosphodiesterase inhibitors. Dev Biol 1996;178:393-402.

18. Wiersma A, Hirsch B, Tsafriri A, Hanssen RG, Van de Kant M, Kloosterboer $\mathrm{HJ}$, et al. Phosphodiesterase 3 inhibitors suppress oocyte maturation and consequent pregnancy without affecting ovulation and cyclicity in rodents. J Clin Invest 1998;102:532-7.

19. Conti M, Andersen CB, Richard F, Mehats C, Chun SY, Horner K, et al. Role of cyclic nucleotide signaling in oocyte maturation. Mol Cell Endocrinol 2002;187:153-9.

20. Jee BC, Chen HY, Chian RC. Effect of a phosphodiesterase type 3 inhibitor in oocyte maturation medium on subsequent mouse embryo development. Fertil Steril 2009;91(5 Suppl):2037-42.

21. Malvezzi H, Sharma R, Agarwal A, Abuzenadah AM, Abu-Elmagd M. Sperm quality after density gradient centrifugation with three commercially available media: a controlled trial. Reprod Biol Endocrinol 2014;12:121.

22. Xue X, Wang WS, Shi JZ, Zhang SL, Zhao WQ, Shi WH, et al. Efficacy of swim-up versus density gradient centrifugation in improving sperm deformity rate and DNA fragmentation index in semen samples from teratozoospermic patients. J Assist Reprod Genet 2014;31:1161-6.

23. Alpha Scientists in Reproductive Medicine and ESHRE Special Interest Group of Embryology. The Istanbul consensus workshop on embryo assessment: proceedings of an expert meeting. Hum Reprod 2011;26:1270-83.

24. Xu KP, Greve T, Smith S, Hyttel P. Chronological changes of bovine follicular oocyte maturation in vitro. Acta Vet Scand 1986;27:50519.

25. Ponderato N, Crotti G, Turini P, Duchi R, Galli C, Lazzari G. Embryonic and foetal development of bovine oocytes treated with a combination of butyrolactone I and roscovitine in an enriched medium prior to IVM and IVF. Mol Reprod Dev 2002;62:513-8.

26. DiLuigi A, Weitzman VN, Pace MC, Siano LJ, Maier D, Mehlmann LM. Meiotic arrest in human oocytes is maintained by a Gs signaling pathway. Biol Reprod 2008;78:667-72.

27. Shu YM, Zeng HT, Ren Z, Zhuang GL, Liang XY, Shen HW, et al. Effects of cilostamide and forskolin on the meiotic resumption and embryonic development of immature human oocytes. Hum Reprod 2008;23:504-13.

28. Jaffe LA, Egbert JR. Regulation of mammalian oocyte meiosis by intercellular communication within the ovarian follicle. Annu Rev Physiol 2017;79:237-60.

29. Adona PR, Lima Verde Leal C. Meiotic inhibition with different cyclin-dependent kinase inhibitors in bovine oocytes and its effects on maturation and embryo development. Zygote 2004;12:197204.

30. Tatemoto H, Terada T. Time-dependent effects of cycloheximide and alpha-amanitin on meiotic resumption and progression in bovine follicular oocytes. Theriogenology 1995;43:1107-13.

31. Kim BK, Lee SC, Kim KJ, Han CH, Kim JH. In vitro maturation, fertilization, and development of human germinal vesicle oocytes collected from stimulated cycles. Fertil Steril 2000;74:1153-8.

32. Nogueira D, Cortvrindt R, De Matos DG, Vanhoutte L, Smitz J. Effect of phosphodiesterase type 3 inhibitor on developmental competence of immature mouse oocytes in vitro. Biol Reprod 2003;69:2045-52.

33. Ola SI, Wang Q, Ai JS, Yin S, Liang CG, Chen DY, et al. Meiotic competence and acetylation pattern of UV light classified mouse an- 
tral oocytes after meiotic arrest with isobutylmethylxanthine. Mol Reprod Dev 2007;74:591-9.

34. De La Fuente R, Eppig JJ. Transcriptional activity of the mouse oocyte genome: companion granulosa cells modulate transcription and chromatin remodeling. Dev Biol 2001;229:224-36.

35. Vanhoutte L, De Sutter P, Nogueira D, Gerris J, Dhont M, Van der Elst J. Nuclear and cytoplasmic maturation of in vitro matured human oocytes after temporary nuclear arrest by phosphodiesterase 3-inhibitor. Hum Reprod 2007;22:1239-46.

36. Son W, Chung J, Cui S, Dean N, Tan S, Chian R. P-704: comparison of embryology and clinical outcome between IVM cycles with and without mature oocytes collected following HCG priming. Fertil Steril 2006;86:S394-S5.

37. Huang JY, Buckett WM, Gilbert L, Tan SL, Chian RC. Retrieval of immature oocytes followed by in vitro maturation and vitrification: a case report on a new strategy of fertility preservation in women with borderline ovarian malignancy. Gynecol Oncol 2007;105: 542-4.

38. Son WY, Lee SY, Lim JH. Fertilization, cleavage and blastocyst development according to the maturation timing of oocytes in in vitro maturation cycles. Hum Reprod 2005;20:3204-7.

39. Dekel N, Lawrence TS, Gilula NB, Beers WH. Modulation of cell-tocell communication in the cumulus-oocyte complex and the regulation of oocyte maturation by LH. Dev Biol 1981;86:356-62.

40. Xie HL, Wang YB, Jiao GZ, Kong DL, Li Q, Li H, et al. Effects of glucose metabolism during in vitro maturation on cytoplasmic maturation of mouse oocytes. Sci Rep 2016;6:20764.

41. Hyttel P, Callesen H, Greve T. Ultrastructural features of preovulatory oocyte maturation in superovulated cattle. J Reprod Fertil 1986;76:645-56. 